\title{
A GESTÃO DE RESÍDUOS SÓLIDOS VISTA A PARTIR DA PRODUÇÃO CIENTÍFICA
}

\section{THE MANAGEMENT OF SOLID WASTE SEEN FROM THE SCIENTIFIC PRODUCTION}

Dulciane Ribeiro Silva ${ }^{1}$, Áquila Priscila Pereira dos Santos ${ }^{2}$, Lucas Braga da Silva $^{3}$, Lucivania Pereira Gloria ${ }^{4}$

\section{RESUMO}

A pesquisa discutiu a gestão de resíduos sólidos a partir da produção científica dos anais da Jornada de Iniciação Científica e Extensão do Instituto Federal do Tocantins. Adotando uma perspectiva de considerar apenas pesquisas empíricas e desenvolvidas neste estado. A pesquisa é de abordagem quali-quantitativa que adotou revisão sistemática nos anais deste evento para a coleta de dados. A análise dos dados coletados aponta que os municípios deste estado enfrentam dificuldades no que diz respeito a infraestrutura institucional e técnica adequada para a gestão de resíduos sólidos. Apresenta ainda a reciclagem, a possibilidade de produzir arte a partir de material obsoleto, gerar renda e reduzir o volume de resíduos depositados no ambiente de modo inadequado, a educação e conscientização ambiental como potencialidades. Nota-se, portanto, que as pessoas físicas e jurídicas de direito público ou privado necessitam adquirir práticas sustentáveis em seu cotidiano para que esta problemática provoque danos ambientais, econômicos e sociais mínimos as sociedades distintas.

Palavras-chave: Resíduos sólidos. Dificuldades. Potencialidades. Produção científica.

\section{ABSTRACT}

The research discusses the solid waste management from the scientific production of the annals of the Journey of Scientific Initiation and Extension of the Federal Institute of Tocantins. Adopting a perspective of considering only empirical researches and developed in this state. The research is a qualitative-quantitative approach that adopted a systematic review in the annals of this event for data collection. The analysis of the collected data indicates that the municipalities of this state face difficulties with regard to the institutional and technical infrastructure adequate for the management of solid waste. It also presents recycling, the possibility of producing art from obsolete material, generate income and reduce the volume of waste deposited in the environment in an inappropriate way, education and environmental awareness as potentialities. It should be noted, therefore, that natural and legal persons governed by public or private law need to acquire sustainable practices in their daily lives so that this problem causes minimal environmental, economic and social damages to different societies.
Graduada em Tecnologia em Logistica pelo Instituto Federal do Tocantins.

E-mail:

dulcianeribeiro4@gmail.com

Graduada em Tecnologia em Logística pelo Instituto Federal do Tocantins.

Mestre em Comunicação e Sociedade pela Universidade Federal do Tocantins.

Mestra em Ambiente e Desenvolvimento pela Universidade do Vale do Taquari.

Keywords: Solid waste. Difficulties. Potentials. Scientific production

$\mathrm{Na}$ atualidade o consumo de materiais é responsável pelo volume cada vez maior de resíduos sólidos depositados e destinados inadequadamente no ambiente. Diante de estímulos específicos os indivíduos se sentem influenciados a consumir e com isso novos tipos de resíduos são gerados. De um lado as práticas de consumo favorecem as empresas 
e de outro geram resíduos e consequências negativas de ordem social, ambiental e econômica (JACOBI; BESEN, 2011; GOUVEIA, 2012).

Para Andrade e Ferreira (2012) os resíduos sólidos provenientes das atividades industriais, domésticas, comerciais, agrícolas, construção civil, saúde, entre outros, resultantes das pressões de mercado e do aumento da população, se não forem adequadamente destinados, podem provocar sérios danos à saúde, ao ambiente, a qualidade de vida das pessoas e até mesmo ao clima.

No que diz respeito aos aspectos legais, a legislação brasileira conta com a Política Nacional de Resíduos Sólidos, dada pela Lei nº 12.305/2010, que disponibiliza aos estados e municípios brasileiros instrumentos e diretrizes para auxiliar os procedimentos de gestão e gerenciamento neste contexto. Além de objetivos específicos, tais como a busca pela proteção da saúde humana, a sustentabilidade, o tratamento e destinação adequada dos resíduos e dos rejeitos (BRASIL, 2010).

Para Jacobi (2006, p. 9) as "dificuldades na gestão dos resíduos sólidos e interferência crescente do despejo inadequado de resíduos sólidos em áreas potencialmente degradáveis em termos ambientais" proporcionam consequências desastrosas em nível nacional, regional e local.

A implementação da política de resíduos sólidos apresenta problemáticas distintas em sua execução nos municípios brasileiros, dentre elas as dificuldades financeiras, orçamentárias, econômicas, institucionais e de recursos humanos qualificados para atuar neste âmbito, principalmente os menores (MAIELLO, 2018).

A gestão e o gerenciamento de resíduos tocantinense apresenta dificuldades de integrar atores estatais e não estatais nas decisões públicas, inexistência de controle social adequado, baixa divulgação das políticas públicas, coordenação interna, fiscalização, regulação e ausência de informações a respeito de linhas de crédito disponíveis (SILVA, 2018).

Além disso, há baixa participação dos representantes políticos nas arenas de debates públicos, ausência de dados específicos que retratem desta questão e dificuldades dos respectivos gestores para desenvolverem pesquisas e projetos para captar recursos (SILVA, 2018).

De acordo com Rolnik (2012, p. 18) a problemática advinda dos procedimentos de gestão e gerenciamento de resíduos sólidos inadequados "é absolutamente urgente, dada a dimensão catastrófica da sua situação nos municípios e nas regiões metropolitanas, e do atraso brasileiro no enfrentamento desse tema". 
Este trabalho está dividido em introdução que consta a delimitação do tema, a problemática, os objetivos da pesquisa, um tópico teórico que retrata da gestão e gerenciamento de resíduos sólidos na contemporaneidade, os métodos de pesquisa utilizados, os resultados e discussões e as considerações finais da pesquisa.

Percebe-se, deste modo, que a preocupação com os resíduos sólidos vem aumentando, e a sociedade em geral deve ser educada de forma, a saber, como descartar os resíduos de forma correta sem agredir o meio ambiente e até mesmo sem prejudicar a própria saúde.

A pesquisa apresentada tem como objetivo discutir a gestão de resíduos sólidos a partir da produção científica dos anais da Jornada de Iniciação Científica e Extensão do Instituto Federal do Tocantins (2013-2018), evidenciando as dificuldades e potencialidades ligadas à gestão de resíduos sólidos dos municípios deste estado.

\section{MATERIAL E METODOS}

A pesquisa desenvolvida objetivou apresentar uma discussão a respeito da gestão de resíduos sólidos a partir de artigos científicos publicados nos anais de um evento realizado anualmente no Tocantins, bem como destacar os estudos de pesquisadores deste estado sobre esta temática a fim de compreender as dificuldades e potencialidades vivenciadas pelos municípios neste contexto.

Adotou-se a revisão sistemática de artigos científicos publicados nos anais da Jornada de Iniciação Científica e Extensão do Tocantins de 2013 a 2018. A escolha deste evento se deu pela abrangência do mesmo e por já ter sido realizado em diversas localidades do estado. Isso possibilita a participação de pesquisadores de instituições de ensino e pesquisa distintas. O estudo se limitou a este período de tempo devido a indisponibilidade dos anais eletrônicos anteriores.

Para Sampaio e Mancini (2007, p. 84) este tipo de estudo "assim como outros tipos de estudo de revisão, é uma forma de pesquisa que utiliza como fonte de dados a literatura sobre determinado tema". Estes mesmos autores consideram que através deste tipo de pesquisa é possível apresentar um resumo com as "evidências relacionadas a uma estratégia de intervenção específica, mediante a aplicação de métodos explícitos e sistematizados de busca, apreciação crítica e síntese da informação selecionada".

Nesta pesquisa, adotou-se abordagem quali-quantitativa, pois houve tanto dados estatísticos; que representam dados expressos em números e também a subjetividade dos pesquisadores na análise das produções científicas (GIL, 2010). 
Tratou-se de uma pesquisa exploratória, tendo em vista que esta possibilitou ao pesquisador, maior contato e número de informações em relação a temática e a problemática pesquisada (GIL, 2010). Ainda, pode ser classificada como básica, pois objetivou "gerar conhecimentos novos úteis para o avanço da ciência sem aplicação prática prevista" (PRODANOV; FREITAS, 2013, p. 51).

A busca de dados compreendeu o período de janeiro a fevereiro de 2019 através de acesso a plataforma eletrônica do evento disponível na internet e as seguintes palavraschave: resíduos sólidos, reciclagem, lixo, logística reversa, embalagens de agrotóxicos e coleta seletiva.

A pesquisa na base de dados identificou 19 pesquisas relacionadas a resíduos sólidos no período apontado. Após a busca de dados foi realizada a leitura dos resumos e dos textos completos. Depois disso, eliminados os artigos teóricos e aqueles que não retratavam de estudos realizados no estado; cerca de 3. Por fim, a análise foi feita de 16 publicações.

A leitura exploratória do material selecionado possibilitou organizar os artigos conforme o quantitativo de trabalhos publicados ligados a este tema, os autores, títulos e ano das publicações, a área de realização do estudo, temática da pesquisa, área em que a pesquisa foi publicada, delineamento do estudo, as dificuldades e potencialidades da gestão de resíduos.

Em um primeiro momento, realizou-se consulta em livros, revistas, artigos, sites oficiais do governo e anais de eventos científicos. Em um segundo momento, da análise nos anais desta jornada para tabulação dos dados e posterior análise.

\section{RESULTADOS E DISCUSSAO}

Aqui é apresentado os resultados e discussões dos dados coletados pela pesquisa. Nota-se, pela análise da tabela 1, que o ano de 2018 representa o maior número de publicações $(37,5 \%)$ do evento ligadas a gestão de resíduos sólidos tocantinense, seguido de 2017 com 18,75\%, 2015 com 18,75\%, 2014 com 12,5\%, além de 2013 e 2015 com um total de $6,25 \%$ respectivamente. 
Tabela 1. Total de publicações por ano e da área de resíduos sólidos

\begin{tabular}{c|c|c}
\hline Edição & Ano & Ligado a resíduos sólidos \\
\hline $4^{\underline{a}}$ & 2013 & $6,25 \%$ \\
\hline $5^{\underline{a}}$ & 2014 & $12,5 \%$ \\
\hline $6^{\underline{a}}$ & 2015 & $18,75 \%$ \\
\hline $7^{\underline{a}}$ & 2016 & $6,25 \%$ \\
\hline $8^{\underline{a}}$ & 2017 & $18,75 \%$ \\
\hline $9^{\underline{a}}$ & 2018 & $37,5 \%$ \\
\hline
\end{tabular}

Fonte: Própria autora (2019)

No quadro 1 é apresentado a edição, os títulos dos trabalhos publicados ligados ao recorte da pesquisa, o ano de publicação e os respectivos autores.

Quadro 1. Edição, título do artigo, ano de publicação e autores ${ }^{1,2,3,4,5,6}$

\begin{tabular}{|c|c|c|c|c|}
\hline$\frac{\varepsilon}{0}$ & 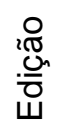 & Título do artigo & $\stackrel{?}{\frac{9}{<}}$ & Autores \\
\hline 1 & $4^{\mathrm{a}}$ & $\begin{array}{l}\text { Impactos dos resíduos dos aparelhos } \\
\text { celulares no meio ambiente e na saúde } \\
\text { humana: percepção de usuários estudantes } \\
\text { de Porto Nacional - TO }\end{array}$ & 2013 & $\begin{array}{l}\text { CASTRO, V. B. de.; LIMA, S. J. de.; } \\
\text { CARVALHO, A. de P.; PARENTE, } \\
\text { R. C. M. }\end{array}$ \\
\hline 2 & $5^{\underline{a}}$ & $\begin{array}{l}\text { Agenda A3P no IFTO e a coleta seletiva } \\
\text { solidária: cooperação para geração de renda } \\
\text { e sustentabilidade ambiental no arranjo } \\
\text { produtivo da coleta seletiva de material } \\
\text { reciclável }\end{array}$ & 2014 & LOPES, C. S. P. \\
\hline 3 & $4^{\mathrm{a}}$ & $\begin{array}{l}\text { Orientação do uso e destinação correta de } \\
\text { embalagens de agrotóxicos na região do bico } \\
\text { do papagaio }\end{array}$ & 2014 & SILVA, R. B. \\
\hline 4 & $6^{\underline{a}}$ & $\begin{array}{l}\text { A dinâmica do lixo em Porto Nacional - TO: } \\
\text { da coleta ao destino final }\end{array}$ & 2015 & $\begin{array}{l}\text { GLORIA, L. P.; SANTOS, L. Q. } \\
\text { dos.; FREITAS, A. S. de }\end{array}$ \\
\hline 5 & $6^{\mathrm{a}}$ & $\begin{array}{l}\text { Logística reversa do lixo eletrônico no } \\
\text { município de Porto Nacional - TO }\end{array}$ & 2015 & 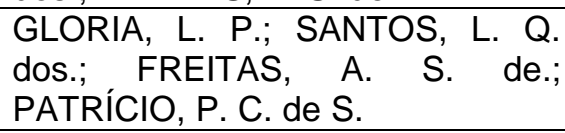 \\
\hline 6 & $6^{\underline{a}}$ & $\begin{array}{l}\text { Logística reversa: reutilização de cones de } \\
\text { linhas de uma empresa de confecção e } \\
\text { serigrafia, destinado ao projeto social AABB } \\
\text { comunidade de Porto Nacional - TO }\end{array}$ & 2015 & $\begin{array}{l}\text { SANTOS, L. Q. dos.; GLORIA, L. } \\
\text { P.; DALL'AGNOL, M.; ALMEIDA, L. } \\
\text { N. A. de }\end{array}$ \\
\hline 7 & $7^{\underline{a}}$ & Compostagem reversa & 2016 & $\begin{array}{l}\text { RÉGO, A. C. G. , LIBÓRIO, R. A.; } \\
\text { MENEZES, A. C. de.; AZEVEDO, } \\
\text { G. J. de.; AZEVEDO, B. da C.; } \\
\text { AZEVEDO, M. da C. A. }\end{array}$ \\
\hline
\end{tabular}

\footnotetext{
${ }^{1}$ http://propi.ifto.edu.br/ocs/index.php/jice/jice/schedConf/presentations (2013)

${ }^{2} \mathrm{http} / / /$ propi.ifto.edu.br/ocs/index.php/jice/5jice/schedConf/presentations (2014)

3 http://propi.ifto.edu.br/ocs/index.php/jice/6jice/schedConf/presentations (2015)

${ }^{4}$ http://propi.ifto.edu.br/ocs/index.php/jice/7jice/schedConf/presentations (2016)

5 http://propi.ifto.edu.br/ocs/index.php/jice/8jice/schedConf/presentations (2017)

${ }^{6}$ http://propi.ifto.edu.br/ocs/index.php/jice/9jice/schedConf/presentations $(2018)$
} 


\begin{tabular}{|c|c|c|c|c|}
\hline 8 & $8^{\underline{a}}$ & $\begin{array}{l}\text { Fortalecendo a Política Nacional de Resíduos } \\
\text { Sólidos por meio da implantação de um } \\
\text { projeto piloto de coleta seletiva e um } \\
\text { programa de educação ambiental no } \\
\text { município de Araguatins }\end{array}$ & 2017 & $\begin{array}{l}\text { SILVA, R. V. S.; SILVA, N. R. da.; } \\
\text { SANTOS, U. G. dos.; SANTOS, M. } \\
\text { A. G. dos.; SILVA, A. V. da }\end{array}$ \\
\hline 9 & $8^{\underline{a}}$ & $\begin{array}{l}\text { Embalagens de agrotóxicos: conscientizando } \\
\text { e destinando }\end{array}$ & 2017 & $\begin{array}{l}\text { SILVA, M. S. da.; TEIXEIRA e } \\
\text { SILVA, A. C.; TORRES, U. A.; } \\
\text { SILVA, S. R. da.; CAMPOS, L. O. }\end{array}$ \\
\hline 10 & $8^{\underline{a}}$ & $\begin{array}{l}\text { Práticas integrativas: avaliação da satisfação } \\
\text { dos agricultores em relação a devolução de } \\
\text { embalagens vazias de agrotóxicos }\end{array}$ & 2017 & $\begin{array}{l}\text { MOTA, M. P.; FERREIRA, P. F.; } \\
\text { GOMES, A. S.; ROCHA, H. } \\
\text { S.;RIBEIRO JÚNIOR, A. A.; } \\
\text { FERREIRA, T. F. }\end{array}$ \\
\hline 11 & $9^{\underline{a}}$ & $\begin{array}{l}\text { Reaproveitamento de resíduos sólidos } \\
\text { orgânicos das escolas por meio da } \\
\text { compostagem em Araguatins - TO }\end{array}$ & 2018 & $\begin{array}{l}\text { SOUZA, R. C. de.; BARBOSA, A. P. } \\
\text { F.; DIAS, J. F. M., FREITAS, I. C. de }\end{array}$ \\
\hline 12 & $9^{\underline{a}}$ & $\begin{array}{l}\text { Resíduos sólidos e sua classificação física: } \\
\text { percepção do técnico em meio ambiente do } \\
\text { câmpus Paraíso/IFTO }\end{array}$ & 2018 & G. da \\
\hline 13 & $9^{\underline{a}}$ & $\begin{array}{l}\text { Pirólise de resíduos sólidos visando produção } \\
\text { de biocombustíveis }\end{array}$ & 2018 & $\begin{array}{l}\text { ALVES, J. V. T. D. A.; SILVA, F. V. } \\
\text { M. da.; PEDROZA, M. M. }\end{array}$ \\
\hline 14 & $9^{\underline{a}}$ & $\begin{array}{l}\text { Construção de placa arduino através da } \\
\text { reutilização de lixo eletrônico }\end{array}$ & 2018 & PEREIRA, C. O.; SOUSA, D. M de. \\
\hline 15 & $9^{\underline{a}}$ & $\begin{array}{l}\text { Reciclagem para contribuir no ensino, } \\
\text { cidadania para interferir no meio ambiente }\end{array}$ & 2018 & SOUSA, R. M. \\
\hline 16 & $9^{\underline{a}}$ & $\begin{array}{l}\text { A utilização da reciclagem como recurso } \\
\text { metodológico no ensino de biologia no colégio } \\
\text { estadual Manoel Vicente de Souza }\end{array}$ & 2018 & $\begin{array}{l}\text { E. C. dos.;, SANTOS, } \\
\text { ERREIRA NETO, B.; } \\
\text { M. A.; NOGUEIRA, M. } \\
\text { O, R. R. do. }\end{array}$ \\
\hline
\end{tabular}

Fonte: Próprios autores (2019)

Percebe-se, pela análise da tabela 2, que a área de realização destas pesquisas corresponderam aos seguintes municípios e localidades: Porto Nacional (25\%), Araguatins (18,75\%), Palmas (12,5\%), Colinas (12,5\%), Augustinópolis $(6,25 \%)$, Gurupi $(6,25 \%)$, Paraíso (6,25\%), Região do Bico do Papagaio (6,25\%), Silvanópolis e Pedro Afonso $(6,25 \%)$. A partir do exposto, nota-se que Porto Nacional teve maior destaque no desenvolvimento de pesquisa neste campo.

Tabela 2. Local de realização do estudo

\begin{tabular}{l|l}
\hline Município do Tocantins & Quantidade \\
\hline Araguatins & $18,75 \%$ \\
\hline Augustinópolis & $6,25 \%$ \\
\hline Colinas & $12,5 \%$ \\
\hline Gurupi & $6,25 \%$ \\
\hline Palmas & $12,5 \%$ \\
\hline Paraíso & $6,25 \%$ \\
\hline Porto Nacional & $25 \%$ \\
\hline Região do Bico do Papagaio & $6,25 \%$ \\
\hline Silvanópolis e Pedro Afonso & $6,25 \%$ \\
\hline Fonte: Próprios autores (2019) &
\end{tabular}


A área de trabalhos de extensão, conforme exposto pela tabela 3, correspondeu ao maior número de pesquisas do recorte temático adotado $(56,25 \%)$, depois as ciências sociais aplicadas (18,75\%), ciências biológicas (12,5\%), exatas e da terra $(6,25 \%)$ e agrárias $(6,25 \%)$. As demais áreas, tais como engenharias, ciências da saúde e humanas, bem como linguística, letras e artes não obtiveram nenhuma publicação. A área de trabalhos de extensão chamou mais a atenção dos pesquisadores que publicaram neste evento.

Tabela 3. Área de pesquisa da publicação ligada a resíduos sólidos conforme especificidades do evento

\begin{tabular}{l|l}
\hline Área da publicação & Quantidade \\
\hline Trabalho de extensão & $56,25 \%$ \\
\hline Ciências exatas e da terra & $6,25 \%$ \\
\hline Ciências biológicas & $12,5 \%$ \\
\hline Engenharias & $0 \%$ \\
\hline Ciências da saúde & $0 \%$ \\
\hline Ciências agrárias & $6,25 \%$ \\
\hline Ciências sociais aplicadas & 3 \\
\hline Ciências humanas & $0 \%$ \\
\hline Linguística, letras e artes & $0 \%$ \\
\hline Fonte: Próprios autores (2019) &
\end{tabular}

De acordo com dados da tabela 4, as temáticas abordadas nas pesquisas foram: embalagens de agrotóxicos (18,75\%), coleta seletiva (12,5\%), resíduos eletrônicos $(12,5 \%)$, compostagem $(12,5 \%)$, logística reversa $(12,5 \%)$, reciclagem $(12,5 \%)$, resíduos sólidos $(6,25 \%)$, coleta e destinação final $(6,25 \%)$ e pirólise de resíduos sólidos $(6,25 \%)$. Os resíduos advindos de embalagens de agrotóxicos representam um maior número de pesquisas desenvolvidas.

Tabela 4. Tema abordado na pesquisa

\begin{tabular}{l|l}
\hline Tema & Quantidade \\
\hline Resíduos sólidos & $6,25 \%$ \\
\hline Coleta seletiva & $12,5 \%$ \\
\hline Resíduos eletrônicos & $12,5 \%$ \\
\hline Coleta e destinação final & $6,25 \%$ \\
\hline Compostagem & $12,5 \%$ \\
\hline Embalagens de agrotóxicos & $18,75 \%$ \\
\hline Pirólise de resíduos sólidos & $6,25 \%$ \\
\hline Logística reversa & $12,5 \%$ \\
\hline Reciclagem & $12,5 \%$ \\
\hline Fonte: Próprios autores (2019) &
\end{tabular}


O delineamento dos estudos, apresentados pela tabela 5 , retratam de pesquisa de campo $(75 \%)$, estudo de caso $(18,75 \%)$ e pesquisa documental $(6,25 \%)$. Nota-se, deste modo, que as pesquisas de campo representam a maioria das pesquisas desenvolvidas.

Tabela 5. Delineamento das pesquisas

\begin{tabular}{l|l}
\hline Tipo de delineamento & Quantidade \\
\hline Estudo de caso & $18,75 \%$ \\
\hline Pesquisa documental & $6,25 \%$ \\
\hline Pesquisa de campo & $75 \%$ \\
\hline Fonte: Próprios autores (2019) &
\end{tabular}

No quadro 1 é possível observar as dificuldades evidenciadas nas pesquisas relacionadas a gestão de resíduos sólidos desenvolvidas no Tocantins.

Quadro 2. Dificuldades da gestão de resíduos sólidos dos municípios do Tocantins

\begin{tabular}{|c|}
\hline Dificuldades \\
\hline $\begin{array}{l}\text { Ausência de coleta seletiva para resíduos eletrônicos de celulares, de pontos de coleta destes } \\
\text { tipos de resíduos e de um aterro sanitário. Além disso, os resíduos são descartados e } \\
\text { incinerados junto com os demais e não existe separação alguma destes ou de outros tipos } \\
\text { (CASTRO et al., 2013). }\end{array}$ \\
\hline $\begin{array}{l}\text { A falta de conscientização ambiental dos alunos de uma instituição de ensino a respeito de } \\
\text { coleta seletiva é percebida como um desafio (LOPES, 2014). }\end{array}$ \\
\hline $\begin{array}{l}\text { Ausência de local adequado para armazenar os resíduos de embalagens de agrotóxicos e de } \\
\text { equipamentos de proteção individual para os profissionais envolvidos neste contexto (SILVA, } \\
\text { 2015). }\end{array}$ \\
\hline $\begin{array}{l}\text { A coleta e destinação dos resíduos sólidos deficientes. Ainda há bastante despejo de lixo em } \\
\text { terrenos baldios e áreas verdes (GLORIA; SANTOS; FREITAS, 2015). }\end{array}$ \\
\hline $\begin{array}{l}\text { Ausência de logística reversa para resíduos eletrônicos e local adequado para o respectivo } \\
\text { descarte (GLORIA et al., 2015). }\end{array}$ \\
\hline letiva e de conhecimentos específicos deste tema e da educação \\
\hline
\end{tabular}

Fonte: Próprios autores (2019)

Verifica-se no quadro 2, as potencialidades da gestão de resíduos sólidos tocantinense a partir das pesquisas publicadas neste evento.

Quadro 3. Potencialidades da gestão de resíduos sólidos dos municípios do Tocantins

\section{Potencialidades}

A logística reversa de cones de linhas de confecção e serigrafia através da produção de artesanato pode ser visto como um meio para reduzir o quantitativo de resíduos do tipo produzidos, gerar renda e contribuir para melhores índices de sustentabilidade (SANTOS et al., 2015).

Adotar a compostagem reversa de resíduos orgânicos para a produção de adubos (RÊGO et al., 2016).

Apresentar a comunidade um ponto de coleta seletiva e a educação ambiental como mecanismos para minimizar os danos causados pelos resíduos produzidos (SILVA et al., 2017). 


\begin{abstract}
Alerta aos agropecuaristas a respeito dos impactos negativos dos resíduos de embalagens de agrotóxicos e a necessidade de destiná-los adequadamente (SILVA et al., 2017).

Aproveitamento de resíduos sólidos orgânicos por meio de compostagem para adubos, além de favorecer a educação e a conscientização ambiental (SOUZA et al., 2018).

Alunos de curso técnico na área ambiental apresentam pensamento teórico-reflexivo a respeito da temática resíduos sólidos (SILVA, 2018).

Apresentar as atividades de reciclagem como uma estratégia metodológica para o ensino e aprendizagem na disciplina de biologia (SANTOS et al., 2018).

A reciclagem como mecanismo para auxiliar no ensino de química (SOUSA, 2018).

A pirólise dos resíduos sólidos para a produção de biocombustíveis (ALVES; SILVA; PEDROZA, 2018).

A construção de uma placa arduíno através dos resíduos eletrônicos (PEREIRA; SOUSA, 2018).

Impacto positivo na educação e conscientização ambiental de agricultores no que diz respeito ao descarte de embalagens de agrotóxicos (MOTA et al. 2017).
\end{abstract}

Fonte: Próprios autores (2019)

Nota-se, portanto, que o evento apresenta maior número de publicações do recorte temático da pesquisa na edição de 2018, os pesquisadores desenvolveram mais pesquisas no município de Porto Nacional e a área de trabalhos de extensão recebeu mais trabalhos do que as demais. A temática que despertou mais interesse das pesquisas foi a relacionada a agrotóxicos e o delineamento de estudo que obteve maior destaque nas pesquisas diz respeito ao estudo de campo.

Pode-se dizer, ainda, que a gestão de resíduos sólidos perpassa dificuldades distintas, tais como a ausência de estrutura institucional e financeira para executar as determinações da política de resíduos sólidos. Além disso, apresenta potencialidades, como a reciclagem, reaproveitamento de materiais consumidos, o desenvolvimento de educação e consciência ambiental.

\title{
4. CONSIDERACOES FINAIS
}

A pesquisa discutiu a gestão de resíduos sólidos a partir da produção científica dos anais da Jornada de Iniciação Científica e Extensão do Instituto Federal do Tocantins, evidenciando as dificuldades e potencialidades da gestão de resíduos dos municípios tocantinenses.

A revisão aponta que o evento publicou mais pesquisas da temática aqui abordada apenas no ano de 2018. Outro ponto a ser destacado, é que os autores das pesquisas preferiram trabalhos na área de extensão e a maior parte dos artigos publicados retratam de Porto Nacional. Os resíduos dos agrotóxicos apareceram em mais pesquisas e o estudo de campo se destacou entre os delineamentos de pesquisa.

Ainda que exista uma política pública que regulamente a problemática de resíduos sólidos e que apresente princípios, objetivos e diretrizes para auxiliar neste sentido, os 
municípios, especialmente os do Tocantins enfrentam dificuldades no cumprimento das exigências desta política.

Dentre as dificuldades, pode-se citar ausência de coleta seletiva de resíduos eletrônicos e de outros tipos nos municípios, ausência de destinação adequada dos resíduos eletrônicos e outros produzidos, de consciência ambiental de alunos, bem como educação ambiental deficiente, falta de local adequado para armazenar resíduos agrotóxicos, despejo de resíduos em locais inadequados.

As potencialidades são distintas, a exemplo, utilizar as práticas de logística reversa para gerar renda e produzir artesanatos, a compostagem de resíduos orgânicos, apresentar a comunidade pontos de coleta seletiva, mostrar os benefícios da educação ambiental para a sociedade, a reciclagem de materiais e alerta a respeito das consequências negativas da produção de resíduos dos mais variados tipos.

Para uma próxima pesquisa científica, sugere-se que seja realizada uma revisão sistemática a respeito da gestão de resíduos sólidos, mas que seja mais abrangente e apresente outros contextos. Através disso ter conhecimento de outras realidades vivenciadas por outros municípios brasileiros para auxiliar e propor novas alternativas para a problemática em questão.

A sustentabilidade da gestão de resíduos sólidos é apresentado pela administração pública como uma premissa necessária para que as gerações do presente e futuro tenham acesso aos recursos naturais e condições de vida equivalentes a de hoje. É necessário que a sociedade adquira práticas sustentáveis em seu cotidiano e que os gestores públicos apliquem os recursos de modo adequado neste sentido.

\section{REFERENCIAS}

ANDRADE, R. M. de; FERREIRA, J. A. A Gestão De Resíduos Sólidos Urbanos No Brasil Frente Às Questões Da Globalização. REDE - Revista Eletrônica do PRODEMA, Fortaleza, v. 6, n. 1, mar. 2011.

BRASIL, Presidência da República do Brasil. Lei no 12.305, de 2 de agosto de 2010. Institui a Política Nacional de Resíduos Sólidos; altera a Lei no 9.605, de 12 de fevereiro de 1998; e dá outras providências. Brasília: 2010.

GIL, A. C. Como elaborar projetos de pesquisa. 5 Ed. - São Paulo: Atlas, 2010.

GOUVEIA, N. Resíduos sólidos urbanos: impactos socioambientais e perspectiva de manejo sustentável com inclusão social. Ciência \& Saúde Coletiva, Rio de Janeiro, v. 17, n. 6, p. 1503-1510, jun. 2012. 
JACOBI, P. Apresentação. In: JACOBI, P. Gestão compartilhada dos resíduos sólidos no Brasil: inovação com inclusão social. São Paulo: Annablume, 2006. p. 9-16.

JACOBI, P. R.; BESEN, G. R. Gestão de resíduos sólidos em São Paulo: desafios da sustentabilidade. Estudos avançados, v. 25, n. 71, p. 135-158, 2011.

MAIELLO, A. BRITTO, A. L. N. de P.; VALLE, T. F. Implementação Da Política Nacional De Resíduos Sólidos. Revista de administração pública, Rio de Janeiro, v. 52, n. 1, p. 24-51, jan./fev. 2018.

PRODANOV, C. C.; FREITAS, E. C.de. Metodologia do trabalho: métodos e técnicas da pesquisa e do trabalho acadêmico. 2 ed. Novo Hamburgo: Feevale, 2013.

ROLNIK, R. RESÍDUOS SÓLIDOS URBANOS: REPENSANDO SUAS DIMENSÕES. In: SANTOS, M. C. L. dos.; DIAS, S. L. F. G. (Orgs.). Resíduos sólidos urbanos e seus impactos socioambientais. São Paulo: IEE-USP, 2012. 82p

SAMPAIO, R. F.; MANCINI, M. C. Estudos de revisão sistemática: um guia para síntese criteriosa da evidência científica. Rev. bras. fisioter., São Carlos, v. 11, n. 1, p. 83-89, jan./fev. 2007.

SILVA, L. B. da. Capacidades estatais para governança de resíduos sólidos no Tocantins. 2018. 111p. Monografia (graduação em administração) - Coordenação do curso de administração, Universidade Federal do Tocantins, Tocantins. 